\title{
Sarcoidosis and autoimmune disorders. A case report and review of the literature
}

\author{
Varan Ozkan 1 \\ Kokturk Nurdan ${ }^{2}$
}

1 Gazi University Faculty of Medicine, Department of Internal Medicine, Division of Rheumatology, Ankara, Turkey

2 Gazi University Faculty of Medicine, Department of Pulmonary Medicine, Ankara, Turkey

Address for correspondence:

Dr. Kokturk Nurdan

Department of Pulmonary Medicine

Gazi University, Ankara, Turkey

E-mail: kokturk.nurdan@gmail.com

\section{Summary}

Sarcoidosis is a multisystem disease of unknown etiology characterized by clusters of non-necrotizing granulomas and distortion of surrounding tissue. Although some patients are asymptomatic, sarcoidosis can cause a wide range of signs and symptoms often mimicking autoimmune rheumatologic disorders. Combined sarcoidosis and autoimmune disorders is uncommon as a result of either an University Hospital database analysis and literature review. However, given the high prevalence of autoimmunity in the general population, clinicians should be alerted to correctly diagnose and manage patients suspected to have both sarcoidosis and autoimmune disorders.

KEY WORDS: sarcoidosis, autoimmunity, connective tissue disease.

Introduction

Sarcoidosis is a systemic inflammatory disorder that involves multiple tissues and is characterized by a non-caseating granulomatous reaction (1). Despite the etiology is not well understood, there are cellular immune system activation and nonspecific inflammatory response similar to some rheumatologic disease which are considered to play an important role in the pathogenesis (2). Sarcoidosis has been reported in all races and ethnic groups with a marked variations. People from different ages can be affected, but it is particularly sighted among the young adults whose ages range from 20 to 40 , with possibly a second peak in women in the seventh decade (1). Sarcoidosis may mimic several systemic diseases displaying different clinical findings, and sometimes has been reported in combination with
Sarcoidosis is a multisystem granulomatous disease that can mimic autoimmune disorders. autoimmune disease (3). Less commonly case of connective tissue diseases (CTDs) have been reported to complicate long-standing sarcoidosis $(3,4)$.

In this mini-review, we briefly present the case of a patient with sarcoidosis and concomitant rheumatoid arthritis. Furthermore, we review the literature on this topic. We searched by Google Scholar and PubMed database the terms <sarcoidosis> and <connective tissue disease $>$ or <autoimmune disorder> .

We also looked for any patient with concomitant diagnosis of sarcoidosis and CTD at the University Hospital of Gazi analyzing its discharge abstracts database. We retrospectively selected patients with the ICD 10 code of D86 admitted to University Hospital of Gazi from 1st Jaunuary 2010 to 31 december 2014. A total of 450 patients with sarcoidosis were identified. Any hospital discharge abstract was evaluated, but only one patient hospital record was identified with concomitant sarcoidosis and rheumatoid arthritis (RA).

\section{Case report}

A 5-year retrospective discharge abstracts review at our University Hospital showed only a single case of concomitant sarcoidosis and autoimmune disease. A 55-year-old woman was diagnosed having sarcoidosis by axillary lymph node biopsy when she was 40year-old. At the time of diagnosis the patient reported tiredness and she showed only lymph nodes enlargement without any lung parenchymal involvement by sarcoidosis at CT scan. After seven years from the diagnosis of sarcoidosis, the patient presented with painful, warm, swollen joints of the both hands and wrists and morning stiffness. At physical examination, several distal and proximal joints were painful and tender. Clinical signs, X-rays and lab tests suggested the diagnosis of active rheumatoid arthritis (RA). In fact, C-reactive protein (CRP) level and erythrocyte sedimentation rate (ESR) were significantly higher than normal. A cyclic citrullinated peptite (CCP) antibody test along with a rheumatoid factor (RF) test confirmed the diagnosis of RA. The patient started a treatment with methotrexate and sulfasalazine with good clinical response. After seven years from the diagnosis of RA, she began to complain pain and redness at 
both legs together with the appearance of multiple skin papules. A skin biopsy revealed noncaseating epithelioid genulomas, consistent with the diagnosis of sarcoid granulomatosis dermatitis. Then, the patient started a treatment with oral prednisolone tablets (initially $0.75 \mathrm{mg} / \mathrm{kg}$ body weight then tapering) showing a complete recovery of both skin lesions and leg sign and symptoms. Recognition of cutaneous sarcoid lesions was important in our patient because they provided a visible clue to the contribution of sarcoidosis for patient symptoms. Nevertheless, the diagnosis of cutaneous lesions in a patient with RA and sarcoidosis may be misleading because lesions can exhibit many different morphologies common to both diseases.

\section{Literature review}

Sarcoidosis is a T helper 1 (Th1) mediated granulomatous disease, with unknown etiology, involving more than one organ. Sarcoidosis may mimic a number of rheumatic diseases or may coexist side by side. It can commonly present with clinical findings that are indicative of vasculitis and spondyloarthritis or connective tissue diseases, including both primary Sjogren's

Syndrome, Systemic Lupus

Excluding Lofgren's and Heerfordt's syndromes, the diagnosis of sarcoidosis requires a tissue biopsy confirming granulomatous inflammation and exclusion of known causes of other granulomatous disease.

Erythematosus, rheumatoid arthritis, and sarcoidosis (1). Some constellations of clinical signs or symptoms are almost pathognomonic for sarcoidosis, including bilateral hilar nodal enlargement as an isolated clinical finding, erythema nodosum with bilateral hilar adenopathy (Lofgren Syndrome), and uveitis combined with fever plus parotiditis (Heerfordt Syndrome). Excluding Lofgren's and Heerfordt's Syndromes, the diagnosis of sarcoidosis requires a tissue biopsy confirming granulomatous inflammation and exclusion of known causes of other granulomatous disease $(5,6)$. Sarcoidosis like CTDs is a multisystem disease that may involve almost any organ system; therefore, it results in various clinical manifestations, sometimes similar to those observed in various CTDs. Nevertheless, the data supporting an association of connective tissue disorders and sarcoidosis are weak in the literature and case reports are few as in our University Hospital database. Judson et al. presented 15 such cases and reviewed 53 cases from the literature. Systemic sclerosis seemed to be the most common type reported as coexisted with sarcoidosis (6). Furthermore, it was determined that the isolated organ involvement of sarcoidosis (37\% of the total cases) could be meaningfully more frequent in the patients who previously diagnosed with connective tissue disease (5). Chopra et al. suggested that the long term connective tissue disease could lead to the granulomatous lesion that could be falsely diagnosed as sar- coidosis but rather a "sarcoid like" granulomatosis inflammation secondary to their long standing connective tissue disorders (6). Therefore, all the alternative diagnosis should be excluded before making the certain diagnosis of sarcoidosis. This is particularly true in patients with a history of connective tissue disorders and previous isolated granulomatosis.

\section{Sarcoidosis and systemic lupus erythematosus}

In 1979 Sharma et al. reported one sarcoidosis patient with systemic lupus erythematosus (7). Later on Begum et al. presented three cases with sarcoidosis and systemic lupus erythematosus (8). Also, one study described 34 patients with sarcoidosis, 10 of them having positive antinuclear antibody (ANA) as measured by Hep2 cell staining, while 2 of them had positive anti-double stranded DNA (anti-dsDNA) antibodies (9). Kobak et al. suggested that the prevalence of positive ANA was significantly higher in patients with sarcoidosis than healthy controls (10). Weinberg et al. (9) concluded that anti-dsDNA may occur in patients with sarcoidosis, but their presence do not predict the subsequent development of systemic lupus erythematosus (SLE).

\section{Sarcoidosis and rheumatoid arthritis}

Sarcoidosis is considered a possible mimic of CTDs, included RA, the most diffuse CTD. Particularly, sarcoidosis and RA may show very similar pulmonary and skin manifestations (Figures 1 and 2), requiring biopsy in case of uncertainty. However the association between sarcoidosis and RA is quite rare in literature reports. Kobak et al. reported 1 patient with sarcoidosis and rheumatoid arthritis. Corticosteroid and methotrexate were started in that case and after sixth month of follow-up, her clinical and laboratory findings and lymphadenopathies on computerized tomography (CT) scan had regressed (11). In 1984 Fallahi et al. and in 1989 Kucera et al. presented 4 patients with sarcoidosis and rheumatoid arthritis $(12,13)$.

\section{Sarcoidosis and systemic sclerosis}

In 1995 Cox et al. reported 7 sarcoidosis patients that have concurrent systemic sclerosis (14). In another report, a patient that have cardiac sarcoidosis with Sjogren's Syndrome, polymyositis and systemic sclerosis have been reported (15). De Bandt et al. reported 5 patients coexistence of sarcoidosis with systemic sclerosis (16). Senda et al. (17) collected 30 consecutive cases of concurrent systemic sclerosis and sarcoidosis (Table 1). 



Figure 1 - CT scan features of various lung involvement in sarcoidosis (left column, yellow letters) and rheumatoid arthritis (right column, light blue letters). A) Conglomerate alveolar masses of sarcoid tissue; B) bilateral peripheral consolidations (organizing pneumonia pattern) in rheumatoid arthritis; C) fibrosing sarcoidosis; D) UIP-like pattern in rheumatoid arthritis.
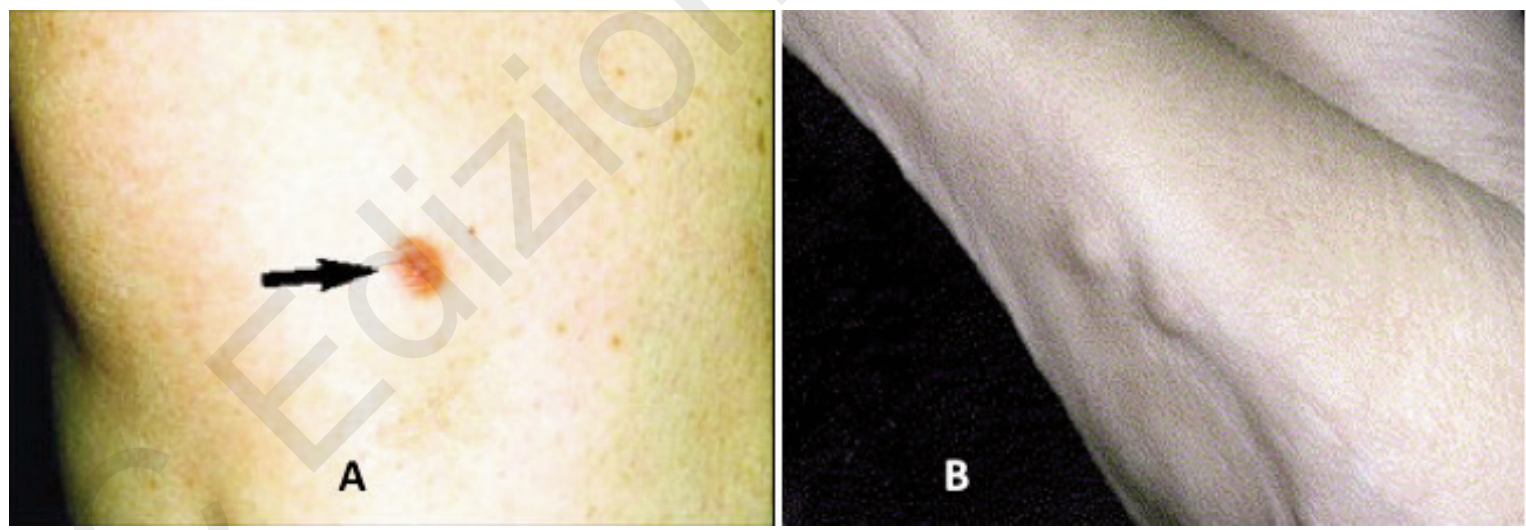

Figure 2 - Some dermatological manifestations in sarcoidosis (left) and rheumatoid arthritis (right). A) Papules of sarcoidosis on the leg; B) rheumatoid nodules under the skin.

Kato et al. (18) and Kobak et al. (19) both presented one case with cutaneous sarcoidosis with concurrent sclerotic fingers of systemic sclerosis. Sakomoto et al. reported a sarcoidosis patient with systemic sclerosis and primary biliary cirrhosis (20).

\section{Sarcoidosis and Sjogren's Syndrome}

In 1979 Sharma et al. reported 3 sarcoidosis patients with Sjogren's Syndrome (7). In a series of 200 patients with sarcoidosis, 4 cases had Sjogren Syndrome (21).
In another series, 5 patients with sarcoidosis were described with concurrent Sjogren's Syndrome (22). Luis et al. presented two cases of sarcoidosis with lung involvement and Sjogren's Syndrome. In that cases, noncaseating granuloma and lymphocytic interstitial pneumonitis were identified in the lung pathology (2325).
Combined pulmonary sarcoidosis and lung involvement of Siogren's Syndrome may require lung biopsy to show noncaseating granulomas and lymphocityc interstitial pneumonitis. 
Table 1 - The characteristics of 30 patients with combined systemic sclerosis and sarcoidosis (17).

\begin{tabular}{ll}
\hline Patients & \# $\mathbf{3 0}$ \\
\hline Female: Male ratio & $21: 9$ \\
Antecedent disease & 16 \\
SSc & 6 \\
Sarcoidosis & 8 \\
Concurrent & \\
Autoantibody & 9 \\
Scl70+ & 8 \\
ACA+ 8 & 7 \\
Scl70/ACA & 6 \\
ND/unclear & 12 \\
SSc classification & 18 \\
Diffuse & \\
Limited &
\end{tabular}

Legend: ACA, anticentromere antibody; ND, not defined; SSc, systemic sclerosis

\section{Sarcoidosis and endocrine autoimmunity}

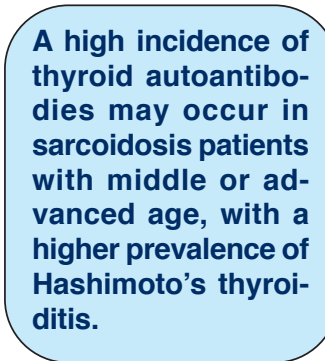

In a case series of 145 sarcoidosis patients, Mayock et al. reported that $2 \%$ of the patients had clinical evidence of thyroiditis. Karlish and MacGregor showed that $1.3 \%$ of the patients with sarcoidosis had Hashimoto thyroiditis (26, 27). Papadopoulos et al.

(28) described 2 patients who had Addison's disease. Among 85 Swedish sarcoidosis patients, thyroid autoimmunity was found in 13 patients including 8 with clinical autoimmune thyroid disease ( 2 with Graves' disease and 6 with autoimmune thyroiditis). Additionally 2 patients had insulin-dependent diabetes mellitus and 1 had premature ovarian failure $(28,29)$. According to a Japanese study, a higher incidence of thyroid autoantibodies may be found in sarcoidosis patients with middle or advanced age and a high prevalence of Hashimoto's thyroiditis (30).

\section{Sarcoidosis and vasculitis}

The coexistence of sarcoidosis and vasculitis has been described elsewhere (31-33). Previously, we have presented a young lady with Lofgren's Syndrome who developed Takayasu arteritis in the follow up period (33). The pathogenetic relationship between sarcoidosis and Takayasu arteritis has not been identified and it is not known if there is a causal relation between the two disorders.

\section{Conclusion}

There are several case reports and few retrospective studies on the association between sarcoidosis and autoimmune disorders.

Sarcoidosis is a well known mimic of several autoimmune diseases, but almost Because of the prevalence of autoimmunity is common in community, the existence of sarcoidosis and autoimmune diseases may be coincidental. uncommonly we can find a combination of sarcoidosis and autoimmune disease in the same patient.

Some reports suggested a significant relationship between sarcoidosis and Sjogren's Syndrome, systemic sclerosis and autoimmune thyroid disease. Judson et al., proposed that the association could develop based on some genetic, environmental and/or immunologic associations $(5,6)$. However there is no epidemiological analysis concerning those associations. Because of the high prevalence of autoimmunity in the community, the co-existence of sarcoidosis and autoimmune diseases may be coincidental. Moreover, the diagnosis of the sarcoidosis might be inappropriate in some CTD cases with granulomatosis. Further studies are needed to put forward this clinical relationship.

\section{References}

1. Valeyre D, Prasse A, Nunes H, Uzunham Y, Brllet PY, Müller-Quernheim J. Lancet. 2013;383:1155-67.

2. Hofmann S, et al. Genome-wide association study identifies ANXA11 as a new susceptibility locus for sarcoidosis. Nat Genet. 2008;40(9):1103-6.

3. Enzenauer RJ, West SG. Sarcoidosis in autoimmune disease. Sem Arthr Rheum. 1992;22:1-17.

4. Pettersson T. Rheumatic features of sarcoidosis. Curr Opin Rheumatol. 1998;10(1):73-8.

5. Judson MA, et al. Concomitant sarcoidosis and a connective tissue disease: review of the clinical findings and postulations concerning their association. Respir Med. 2013;107(9):1453-9.

6. Chopra A, Judson MA. How are cancer and connective tissue diseases related to sarcoidosis? Curr Opin Pulm Med. 2015;21(5):517-24.

7. Wiesenhutter GW, Sharma OP. Is sarcoidosis an autoimmune disease?: Report of four cases and review of the literature. Semin Arthritis Rheum. 1979;9(2):124-44.

8. Begum S, et al. Concurrence of sarcoidosis and systemic lupus erythematosus in three patients. Clin Exp Rheumatol. 2002;20(4):549-52.

9. Weinberg, I, Vasiliev L, Gotsman I. Anti-dsDNA antibodies in sarcoidosis. Semin Arthritis Rheum. 2000; 29(5):328-31.

10. Kobak S, et al. The prevalence of antinuclear antibodies in patients with sarcoidosis. Autoimmune Dis. 2014;2014:351852.

11. Kobak S, et al. Co-occurrence of rheumatoid arthritis and sarcoidosis. BMJ Case Rep. 2015;2015. 
12. Fallahi $\mathrm{S}$, et al. Coexistence of rheumatoid arthritis and sarcoidosis: difficulties encountered in the differential diagnosis of common manifestations. J Rheumatol. 1984;11(4):526-9.

13. Kucera RF. A possible association of rheumatoid arthritis and sarcoidosis. Chest. 1989;95(3):604-6.

14. Cox D, Conant E, Earle L, et al. Sarcoidosis in systemic sclerosis: report of 7 cases. J Rheumatol. 1995;22(5):881-5.

15. Hosoya N, et al. A rare case of cardiac sarcoidosis in a patient with progressive systemic sclerosis, Sjogren's syndrome, and polymyositis. Intern Med. 1995;34(12):1164-7.

16. De Bandt M, et al. Systemic sclerosis and sarcoidosis, a report of five cases. Br J Rheumatol. 1997;36 (1):117-9.

17. Senda S, Igawa K, Nishioka M, et al. Systemic sclerosis with sarcoidosis: case report and review of the published work. J Dermatol. 2014;41(5):421-3.

18. Kato Y, Ohtsuka M, Yamamoto T. Cutaneous sarcoidosis concurrently involved in the sclerotic fingers of a patient with systemic sclerosis. J Dermatol. 2015;42(3):331-3.

19. Kobak S, Sever F, Sivrikoz O, Karaarslan A. Coexistence of sarcoidosis and systemic sclerosis. Case Rep Rheumatol. 2013;2013:ID 684216.

20. Sakamoto N, Ishimatsu $Y$, Kakugawa T, et al. Sarcoidosis in a patient with systemic sclerosis and primary biliary cirrhosis. Intern Med. 2010;49(15):160911.

21. Sharma OP. Sarcoidosis and other autoimmune disorders. Curr Opin Pulm Med. 2002;8(5):452-6.

22. Ramos-Casals M, et al. Sarcoidosis or Sjogren syndrome? Clues to defining mimicry or coexistence in 59 cases. Medicine (Baltimore). 2004;83(2):85-95.
23. Lois M, et al. Coexisting Sjogren's syndrome and sarcoidosis in the lung. Semin Arthritis Rheum. 1998;28 (1):31-40.

24. Mansour MJ, Al-Hashimi I, Wright JM. Coexistence of Sjogren's syndrome and sarcoidosis: a report of five cases. J Oral Pathol Med. 2007;36(6):337-41.

25. Gal I, Kovacs J, Zeher M. Case series: coexistence of Sjogren's syndrome and sarcoidosis. J Rheumatol. 2000;27(10):2507-10.

26. Mayock RL, et al. Manifestations of Sarcoidosis. Analysis of 145 Patients, with a Review of Nine Series Selected from the Literature. Am J Med. 1963;35:67-89.

27. MacGregor GA. Sarcoidosis, thyroiditis, and Addison's disease. Lancet. 1970;2(7685):1257.

28. Papadopoulos $\mathrm{KI}$, et al. High frequency of endocrine autoimmunity in patients with sarcoidosis. Eur J Endocrinol. 1996;134(3):331-6.

29. Isern V, et al. Sarcoidosis and autoimmune thyroid disease. A case series of ten patients. Sarcoidosis Vasc Diffuse Lung Dis. 2007;24(2):148-52.

30. Nakamura $\mathrm{H}$, et al. High incidence of positive autoantibodies against thyroid peroxidase and thyroglobulin in patients with sarcoidosis. Clin Endocrinol (Oxf). 1997;46(4):467-72.

31. Wang LW, et al. Granulomatous sarcoid aortitis: a serious complication of a well-known multisystem disease. Lancet. 2015;385:2014.

32. Espinoza GM, Deasai A, Akduman L. Ocular Vasculitis. Curr Rheumatol Rep. 2013;15(9):355.

33. Kokturk N, Turktas H, Ozturk MA, Aksoy H, Atasever T. The Role of Positron Emission Tomography for the Diagnosis and Follow up of a Patient with Sarcoidosis and Takayasu Arteritis. South Med J. 2007; 100(3):331. 